\title{
The analysis of improving effect of parallel hole cut blasting on the basis of test results
}

\author{
Xingjun Fan 1, a Bin Chen ${ }^{2, b}$, Jinxi Duan $3, \mathrm{c}$ \\ 1,2,3. College of Basic Education, National University of Defense Technology, Changsha, Hunan, \\ 410072, China \\ a724072166@qq.com, bchenbin_gfkd@sina.com, c13677303877@163.com
}

\begin{abstract}
Keywords:parallel hole cut blasting, spacing between empty hole and blast hole, hole blocking Abstract. In order to realize the fast excavation in hard rock roadway with large footage, according to experience and parallel hole cut blasting theory, a diamond central interval charging parallel hole cut blasting experiment scheme was designed, and a series of test was finished in Beishan test site. But through the measurement and evaluation of the test results, it is found that the blasting effect is not ideal. Through research and analysis, It is concluded that the spacing between empty hole and blast hole has an important influence on cut blasting effect, drilling quality will seriously affect the cut blasting effect and a good hole blocking and reasonable jam length play an important role to improve cut blasting effect.
\end{abstract}

\section{Introduction}

In tunneling blasting engineering, directly disposed charge hole blasting are less effective since only one free surface is existed, often appear "cannon rushing" phenomenon, or low footage, the high rate of residual hole, hole utilization rate is low ${ }^{[1]}$. Especially for the blasting excavation of hard rock tunnel with small cross-section, direct arrangement charging hole blasting doesn't generally make a good blasting effect due to the small section and strong rock clip force, rock block is big, secondary blasting and slag and other auxiliary operation time are increased, and the influence on the surrounding rock stability is increased. So in tunnel excavation blasting, generally, in order to achieve good blasting effect, cut holes and auxiliary holes, perimeter holes, caving holes and bottom holes are generally set, different holes play different roles, they need to cooperate and complement each other.

Because only one free surface is existed, the rock initial clamping action is big, and the blasting is more difficult. In order to increase more free surface and reduce the difficulty of blasting excavation of tunnel, a small amount of holes are usually decorated in the proper position on the surface. These blasting holes will be detonated in advance, which will lead to a relatively small volume of initial groove, which will provide more free surface and expansion space for the subsequent blasting hole. The subsequent initiation holes that offers second free surface and broken rock expansion space are called cut holes.

\section{Important influence factors of cut blasting}

Cutting blasting is the key to the blasting excavation of rock tunnel, and the main factors influencing the blasting effect are the cutting method and blasting parameters. Cutting blasting is a physical and chemical process of rock and explosive, which is accompanied by high temperature, high pressure and high strain. There are four main factors influencing the blasting effect of cut blasting: explosive properties, rock properties, cutting blasting parameters and construction technology ${ }^{[2]}$. These four factors are interrelated, mutual influence and mutual restriction. In the design of cutting blasting, first of all, according to the actual rock properties of the blasting site, it is appropriate to choose appropriate explosives and determine the reasonable cutting blasting parameters, and choose the correct construction technology, which can achieve a more ideal cut blasting effect.

Rock properties. It is generally believed that the failure of rock under explosion is the result of the interaction of stress wave and explosion gas expansion. According to wave impedance of rock, the rock is divided into three categories: rock with low wave impedance and rock with medium wave impedance and rock with high wave impedance ${ }^{[3]}$. It is considered that the peak value of stress wave 
is different when the rock is damaged with different wave impedance. For the rock with high wave impedance, the rock failure is mainly caused by the stress wave generated by the explosion of the explosive. For the rock with medium wave impedance, rock failure is due to the explosive stress wave and explosive gas expansion effect. For the low wave impedance rock, the expansion of the detonation gas plays an important role on the failure of rock.

On the other hand, the rock is a typical brittle material, and structural defects such as cracks, joints and faults are generally existed.And its performance to structural defects is very sensitive, rock mass structure characteristics will seriously affect the cutting blasting effect. The structural defects in rock mass have positive effect on the crack propagation and extension. The stress concentration effect can be produced by the rock mass in the structure defect, which is beneficial to the rock fragmentation. The formation of rock burst fracture zone first appeared in the structural defects. However, if the structural defects in the rock mass is too much, it will cause the explosion of the premature escape of gas, resulting in energy loss.Due to the changeable geological condition in rock tunnel excavation blasting and rock properties are different, so in rock tunnel blasting design of coping rock properties to judge and measure, so as to select the appropriate cut blasting method and the construction technology.

Explosive properties. Rock blasting fragmentation mainly depends on the energy released by the explosive, the main parameters affecting the blasting effect are density, detonation and detonation velocity.In rock blasting, explosion pressure is greater, more intense damage to the rock and throwing action. The volume of detonation temperature, explosion and explosion gas has great influence on the explosion pressure. It is an effective way to improve the blasting effect by using the high density and the larger power of the explosive.

Another important parameter that affects the performance of the explosive is detonation velocity, and the explosive stress wave with different detonation velocity can be excited by different parameters in rock, which has important influence on the blasting effect.In the same case of the same explosive density and detonation heat, the higher the detonation velocity, the greater the peak stress wave generated in the rock, but the corresponding reduction in the time of action. Therefore, in rock blasting, rock crack growth and fracture is not only determined by the peak of the explosion stress wave, but also with the stress time, the stress wave shape and other factors.Therefore, in order to obtain better blasting results and reduce the consumption of explosives, the explosive used in the rock must be selected according to the rock properties. In general,high strength explosive are selected for high impedance rocks, and high explosive are selected for low wave impedance rocks ${ }^{[4]}$.

Cut blasting parameters. Cutting blasting parameters are the key factors influencing the blasting effect. For different construction conditions and geological conditions of rock mass, different engineering survey and section size, the targeted design of blasting parameters is required. For the parallel hole cut blasting, according to the engineering experience summary there are three ways of cutting : cracks cutting, barrel cutting and the spiral slotting. Three cutting methods have their own advantages and disadvantages, which need to be selected according to the different engineering survey and rock properties.In addition, the design of blasting parameters include explosive ratio, hole diameter, hole depth, hole spacing etc. The different parameters are interrelated and influence each other, so it is needed to determine the reasonable blasting parameters according to the actual situation to get a better effect.

Construction technology. With the development of blasting equipment and drilling tools, the influence of construction technology on the blasting effect is more and more important. parallel hole blasting has higher requirements on the accuracy of drilling, which requires drilling construction technicians have a higher professional standards. Due to the uneven level of drilling technical personnel, the quality of drilling is not satisfied with the requirements, this kind of situation often happens.In addition to the impact of the cutting effect of the construction process also includes supporting methods, base station structures, blasting hole blocking, etc.. Selection of applicable construction equipment and scientific construction method is an important measure to ensure good cutting effect. 


\section{Test plan}

The engineering overview of test site. In the Beishan test site, traffic is convenient. The surrounding rock is mostly granite, and rock texture is relatively hard. Coupled with smaller section and the strong rock clamping effect, the ordinary way of blasting hole remains high rate of residual holes, circulating footage is small and the tunneling rate is low. In order to improve the blasting effect and increase circulation footage, according to the actual situation of engineering, the diamond in the middle of interval charging of parallel hole cut blasting method is designed. Air leg drill model is YT-28 in test, drill diameter is $38 \mathrm{~mm}$, hole diameter is $40 \mathrm{~mm}$, using $2 \#$ rock ammonium nitrate explosive. The specification of explosives is $32 \mathrm{~mm} \times 200 \mathrm{~mm}$.

Layout of blasting holes and parameters of blasting holes in cut blasting. The parallel hole cut blasting is not affected by the size of the section, in the medium and small section rock tunnel blasting, parallel cut blasting method is generally chosen. But parallel hole blasting has higher requirements on the accuracy of drilling, which requires that the hole is strictly parallel. A certain number of empty holes are often arranged in the parallel hole blasting, and the free surface effect and pressure relief effect are used to provide second free surface and rock compensation space for the cut blasting. In this experiment, we used the diamond cut blasting method, set up 4 empty holes and 5 charge holes, and then, based on the method of the middle interval charge. Simplified model of parallel hole cut blasting with interval charge at the middle part is shown in Fig 1. Borehole layout and hole parameters is shown in Fig 2.

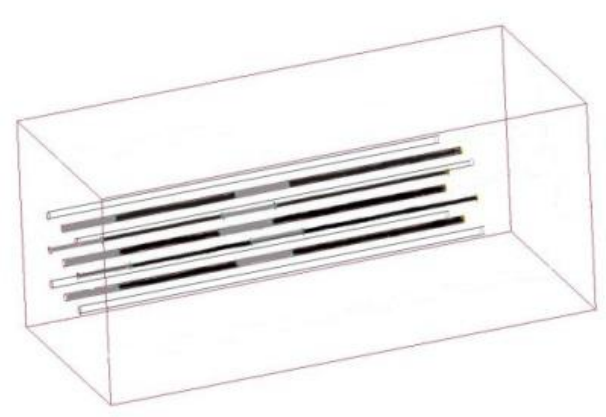

Fig.1 Simplified model of parallel hole cut blasting with interval charge at the middle part

\section{Test process and result analysis}

Drill holes. Drilling hole is an important part of the parallel cut blasting, and good drilling accuracy is the key to ensure the blasting effect. In the installation of drilling equipment, to ensure that the drilling tool is installed correctly. Professional drilling technicians are selected as far as possible, and the same drilling technical personnel were selected in the same cycle, which avoids the influence of human factors on the drilling accuracy. The drilled holes are shown in Fig.3.

Explosive charge. The charge method is the middle interval charge in this test, and the charge structure is the coupling charge. In the parallel cut blasting of the hard tunnel, the coupling charge is generally adopted, which can make full use of the explosive stress wave breaking rock. It is important to ensure the proper installation of explosives when loading explosives, a big gap can not be existed between adjacent drug volume, explosive can not be vigorously loaded to avoid excessive deformation of the drug volume. The blasting hole after loading is shown in Fig.4. 


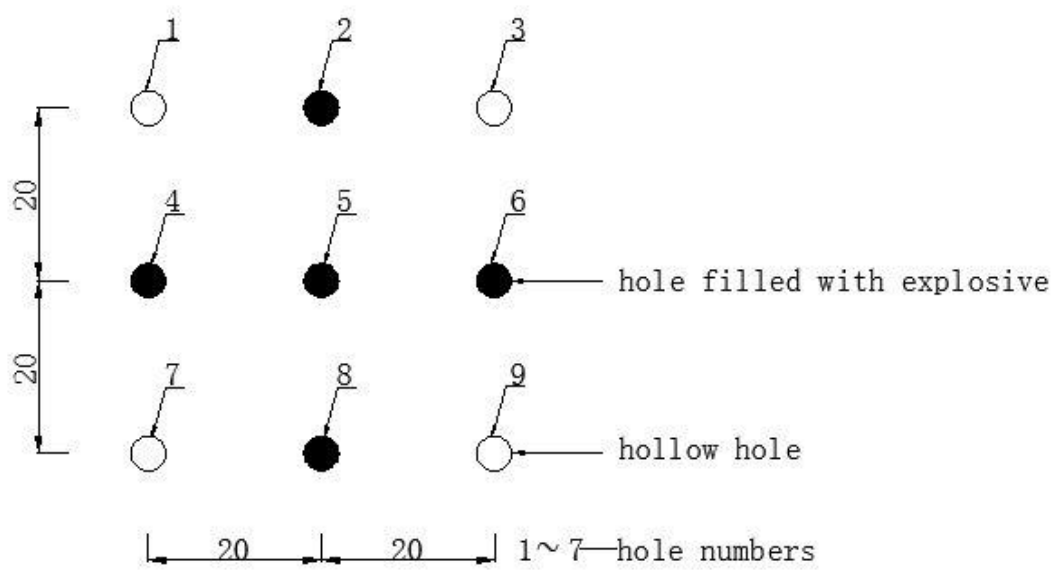

(a) holes arrangement layout

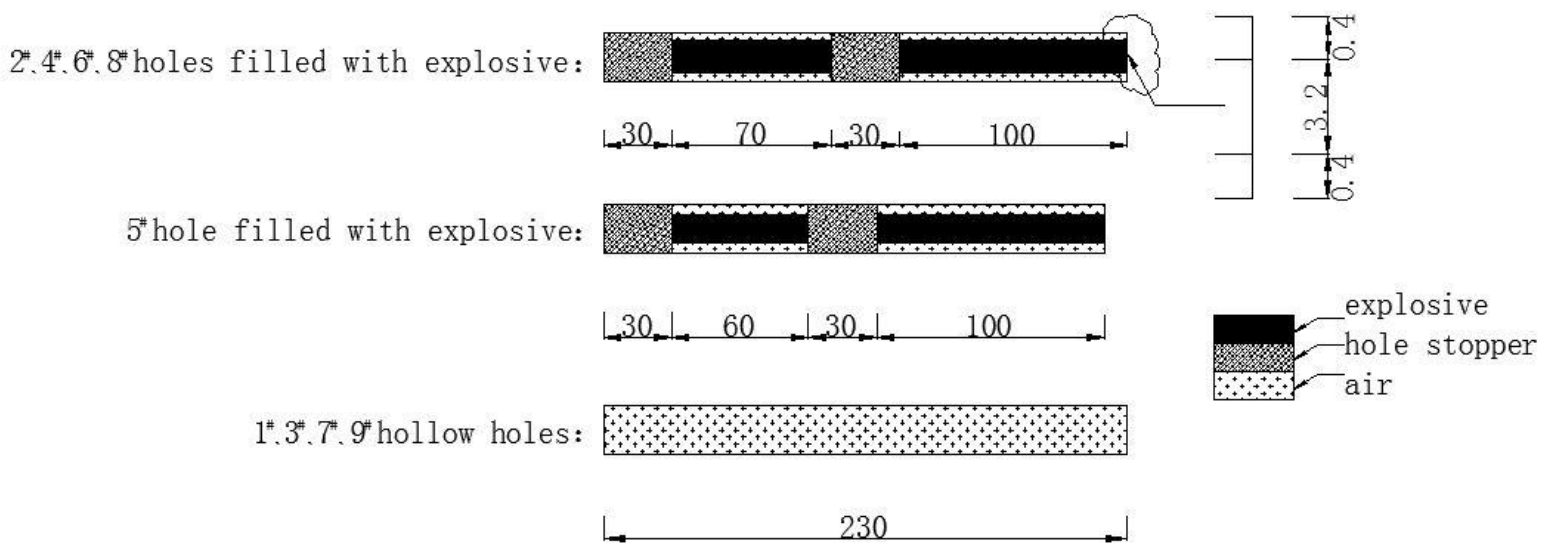

(b) structural profiles of holes

Fig.2 Borehole layout and hole parameters

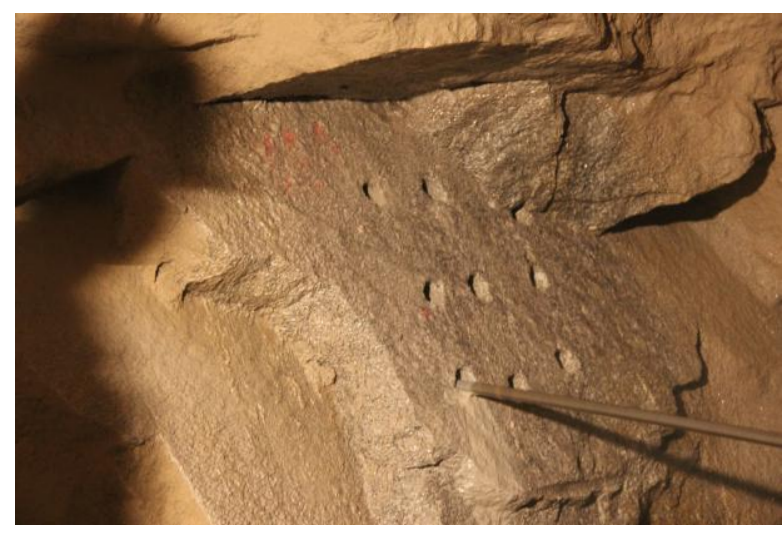

Fig.3 Graphics for the drilled holes

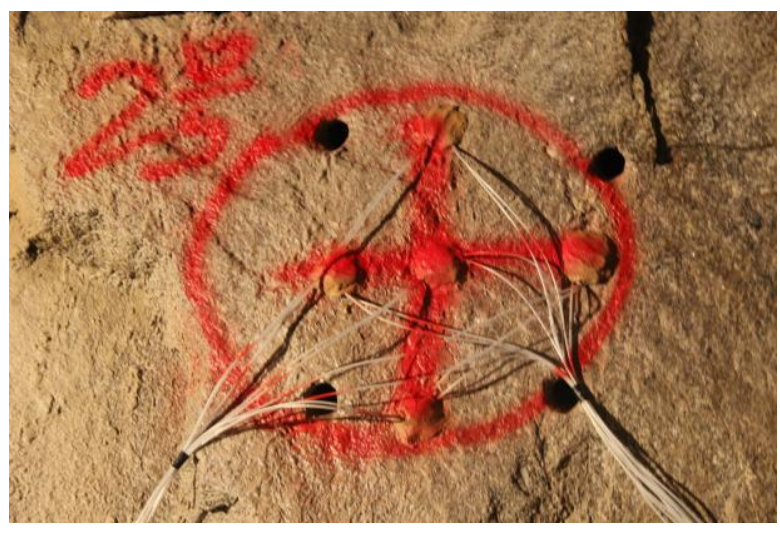

Fig.4 The blasting holes after loading

Initiation.There are two kinds of initiation modes: reverse initiation and forward initiation. In most cases the reverse initiation mode is better than the forward initiation. Reverse initiation method can prolong the explosive gas in the inner hole of the time and to prevent gas premature explosion from escaping from the orifice, resulting in loss of energy. This test adopts the way of the middle interval charge, the two stage of explosive is detonated at the same time, and there was no delayed initiation. The network should be carefully checked before the initiation of initiation, and ensure the safety of the perimeter.

Analysis of blasting effect.After blasting, the blasting results are measured and evaluated. The results show that the blasting effect is not ideal, which shows that the volume of the groove is smaller 
and the depth of the groove is smaller, the length of the residual hole is larger, and the blasting effect is shown in Fig.5.

Through the analysis of the construction method and the cutting parameters, it can be known that there are three main reasons for the unsatisfactory of the cut, the large spacing between blast hole and the empty hole, the hole deviation and the poor obstruction.

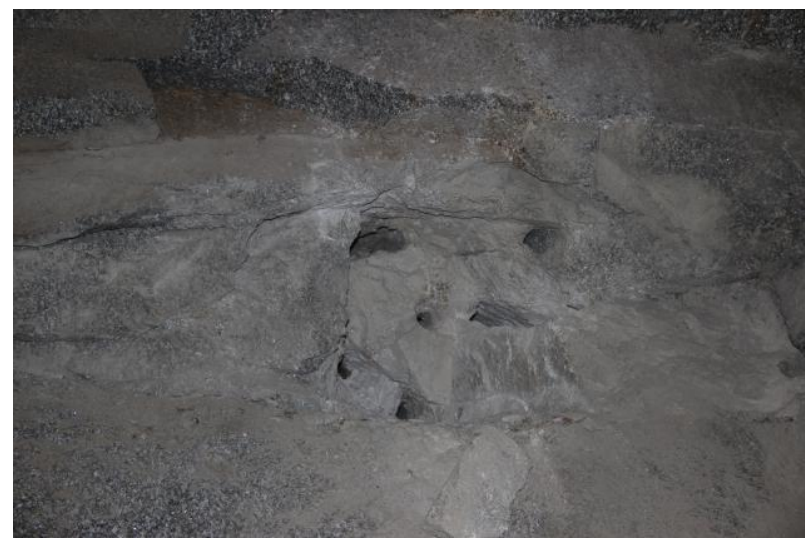

(a)

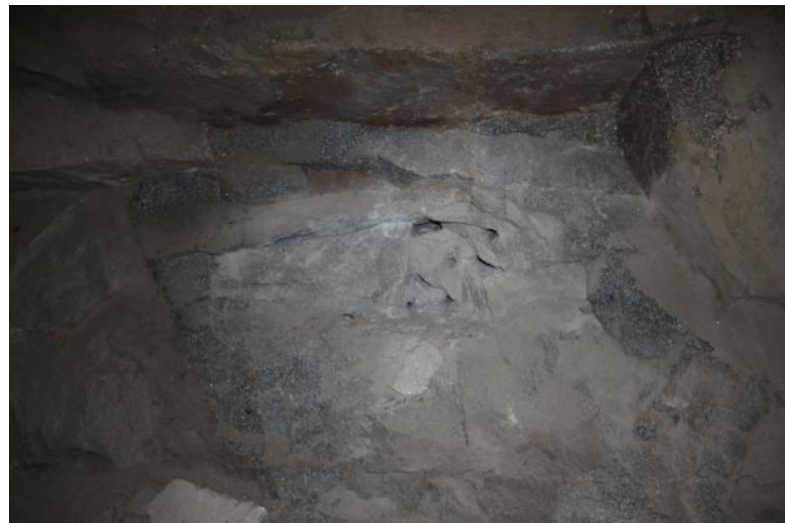

(b)

Fig.5 Sketch pictures of cut blasting effect

\section{Conclusions}

In the actual engineering construction, on the one hand, the professional technical personnel are required and it is necessary to use specialized drilling equipment to ensure the drilling accuracy as far as possible. On the other hand, according to the practical experience of the project, the deviation of drilling hole is difficult to avoid in the engineering construction. Therefore, in order to avoid the occurrence of this kind of situation, in the case of blasting design, the distance between the charging hole and the empty hole is smaller than the radius of the rock crushing zone, the breaking of rocks is the result of the explosion stress wave, the main function of explosion gas is to throw the rock fragments, and in the rock cracking explosion gas does not play a leading role. In this case, if the hole bottom distance is small due to the deviation of the drilling hole, the rock can be broken under the action of blast stress wave, and the effect of the gas explosion is to throw the broken rock out of the cavity. Due to the explosion of gas is not involved in the fragmentation of the rock, even if the hole bottom runs through leading to the explosion gas leakage will not affect the cutting effect. In another case, if the hole bottom distance is large due to the deviation of the drilling hole, the distance is likely to remain in the fracture zone, explosion gas is involved in the fragmentation of rock, and the rock burst under the combined action of blast stress wave and explosion gas, and the cracked rock was thrown out of the cavity ${ }^{[5]}$. Therefore, the method is to improve the success rate of the cut, to improve the effect of the cut, to avoid the hole deviation caused by the failure of the effective way of cutting.

In tunnel and tunnel engineering blasting, blasting hole blocking is an important factor to influence the blasting effect. Hole blocking body (stemming) closed hole, to prevent detonation gas prematurely from escaping from the orifice caused by pressure relief, resulting in the loss of energy. The other function of the hole blocking body (stemming) is to reduce the pressure and the temperature of escaping gas from the hole, and to prevent the explosion of the explosion products such as the gas or dust. In addition, the hole blocking body (stemming) also plays a role in increasing the effective destruction of the explosive medium, changing the form and scope of the destruction of the explosive medium, and so on ${ }^{[6]}$. 


\section{References}

[1] Shihai Chen, Haixia Wai, Aizhi Xue. Experimental study of the deep hole cut blasting in hard rock tunnel[J]. Chinese Journal of Rock Mechanics and Engineering, 2007, 26(s1): 3498-3502.(in Chinese)

[2] Qi Zhang, Yongqi Yang, Yongfeng Yuan, et a1. Study on the factors affecting parallel hole cut blasting[J]. Rock and Soil Mechanics, 2001, 22(2): 144-147.(in Chinese)

[3] Dianshu Liu, Bin Yu, Yulan Du,et al.Damage models of rock fragmentation by blasting and their progress[J]. Engineering Blasting, 1999, 5(4):78-84.(in Chinese)

[4] Renliang Shan, Jijun Zhou, Baolong Huang. Analysis on slotting blasting influence factors of mine roadway [J]. Coal Science and Technology, 2010, 38(2): 25-27.(in Chinese)

[5] Weidong Duan, Shigao Hu. The best choice of empty blasting hole spacing in the presplit blasting[J]. Mineral Engineering Research, 2012, 27(1): 4-7.(in Chinese)

[6] Yong Luo, Zhaowu Shen. Study on length of stemming material and its effect in hole-charged blasting[J]. Mechanics in Engineering, 2006, 28(2): 48-52.(in Chinese) 